\title{
Modelling of the beam-plasma interaction in a strongly inhomogeneous plasma
}

\author{
Aleksander Volokitin ${ }^{* \dagger}$, Vladimir Krasnoselskikh, ${ }^{\dagger}$, Catherine Krafft** and Evgenii \\ Kuznetsov ${ }^{\ddagger \dagger}$
}

*IZMIRAN / IKI, RAS, Russia

${ }^{\dagger}$ LPC2E, CNRS-University of Orléans, France

${ }^{* *}$ LPP, Ecole Polytechnique, Univ. Paris-Sud, France

${ }^{\ddagger}$ P.N. Lebedev Institute of Physics, RAS

\begin{abstract}
The interaction of a beam propagating in a inhomogeneous solar wind plasma is considered. The properties of the plasma waves are described by the one dimensional Zakharov's equation, the beam is modelled by means of particles moving in the electric fields of the Langmuir waves. We take into account the presence of high level density fluctuations that are known to be present in the solar wind. It is shown that when the level of density fluctuations is low, $\delta n / n_{0} \ll 3 k^{2} \lambda_{d}^{2}$, the regime of beam relaxation is very similar to that occurring in a homogeneous plasma and can be described by the quasilinear (QL) equations. In this case, the relaxation length is very short and corresponds to that obtained using the QL approximation. On the contrary, when the level of density fluctuations overcomes some limit, i.e. $\delta n / n_{0} \geq 3 k^{2} \lambda_{d}^{2}$, where $\delta n / n_{0} \ll 1$, the plasma inhomogeneities crucially influence the process of relaxation. First, the linear wave growth becomes localized and clearly identifiable wave packets/clumps dominate the wave spectrum; this is associated with the kinematic properties of the waves' propagation and the wave-particle resonant interactions. Most of the wave packets grow in the regions of density gradients. The second important feature revealed by the beam relaxation consists in the generation of a tail of accelerated electrons with velocities $V>V_{b}$ exceeding the beam drift. The beam widens in both directions, toward lower velocities as well as higher velocities; the density of the accelerated electrons can reach more than $10 \div 20 \%$ of the beam density and the energy flux carried by this population can become as large as $40 \%$ of the initial energy flux of the beam.
\end{abstract}

Keywords: <Langmuir wave, turbulence>

PACS: 52.35.Ra; 52.65.-y

\section{MOTIVATION}

Since the beginning of space research, Langmuir waves have been the subject of intensive studies in the solar wind and, in particular, in the regions of the electron foreshock of the Earth and of other planets. These waves are generated during the beam-plasma interaction. Initially, experimental studies of such processes were carried out in the source regions of the type III solar radio bursts. Direct in situ measurements at 1 AU have shown the simultaneous occurrence of bump on tail electron distributions and plasma wave energy growth above the background thermal noise [1]. No any plateau-type particle distributions as predicted by the quasilinear theory were observed. It was also pointed out that plasma waves were clumped into spikes with peak amplitudes typically three orders of magnitude above the mean [2]. Some authors [3], analyzing Langmuir waves in type III solar radio bursts sources, came to the conclusion that no evidence exists in the experimental data of any strong nonlinear phenomena such as soliton formation or collapse. They proposed an explanation of the clumping phenomenon based on the idea that the plasma is inhomogeneous, and that in most of the regions where the beam could ex- cite the waves the characteristic scale of the inhomogeneity was comparable with the spatial growth rate, thereby resulting in the suppression of the instability. They argued that sufficient amplification occurs only along certain paths where, by chance, successive inhomogeneities are sufficiently similar not to interfere with the amplification process leading to the observed spikes. This idea was further developed by [4]-[5] and [6]-[7]. A very similar problem of beam-plasma interaction was studied in laboratory plasmas. The effects of density fluctuations were found to be important for the development of the instability. Assuming the amplitude of density fluctuations to be sufficiently large, some authors [8]-[9] have described the evolution of the beam in the quasilinear one dimensional approximation and noticed two important effects that are distinct from the case of beam-plasma evolution in a homogeneous plasma. The first consists in the locking of the instability even when the beam velocity distribution still presents a positive slope, and the second in the formation of a tail of energetic electrons due to the variations of the phase velocities of waves moving along the inhomogeneous density profile.

The observations of large scale electron density fluctuations in the solar wind gave strong arguments in fa- 
vor of such an interpretation. In situ measurements of the density fluctuations' spectrum by ISEE 1 and 2 satellites [10] revealed that characteristic density fluctuations as large as $\delta n / n>10^{-2}$ may exist on a scale of the order of $100 \mathrm{~km}$, while interplanetary scintillation measurements from extragalactic radio sources give an average value around $\delta n / n \sim 10^{-3}$ [11]. Some authors [10] also argued that the corresponding power index for high frequencies is variable, while for low frequencies it is approximately constant, and the absolute value of density fluctuations is proportional to the mean plasma density. The main contribution to the fluctuations' level comes from the high frequencies, and in the range $4-16 \mathrm{~Hz}$ the mean relative fluctuation can be as large as 0.04 . In addition, no evidence was found [12] of a strong anisotropy of density fluctuations. More recently, density fluctuations' spectra have been deduced from the EFW probe potential variations measured onboard the Cluster spacecraft in the free solar wind [13]. Recently, simulations of the beam-plasma interaction in the presence of density fluctuations and monotonously decreasing density profiles similar to heliospheric conditions have been carried out [14]. They have confirmed that the beams can indeed propagate over quite long distances in the solar wind and they clearly showed the formation of tails of energetic electrons. Recently, some authors [15]-[16] reported simulation results on the beam plasma instability for several pre-selected density profiles, illustrating the effects of wave clumping and trapping.

Hereafter we describe the model that we developed to study the beam-plasma interaction in inhomogeneous plasmas with quasi-random density fluctuations. Some relevant simulation results are also shown.

\section{D MATHEMATICAL MODEL}

In order to describe the Langmuir turbulence and the beam interaction with a plasma presenting background density fluctuations we use the 1D Zakharov's equations

$$
\begin{gathered}
\left\{i \frac{\partial}{\partial t}+\frac{3 T_{e}}{2 m_{e} \omega_{p}} \frac{\partial^{2}}{\partial z^{2}}\right\} E=\frac{\omega_{p}}{2} \frac{\delta n}{n} E, \\
\frac{\partial}{\partial t} \frac{\delta n}{n}=\frac{\partial}{\partial z} v_{i}=\frac{\partial^{2}}{\partial z^{2}} \Phi, \\
\frac{\partial v_{i}}{\partial t}=\frac{\partial}{\partial z} \frac{\partial \Phi}{\partial t}=-\frac{\partial}{\partial z}\left(\frac{T_{e}+3 T_{i}}{m_{i}} \frac{\delta n}{n}+\frac{|E|^{2}}{16 \pi m_{i} n}\right),
\end{gathered}
$$

where the electric field is $\mathscr{E}=\operatorname{Re}\left(E e^{-i \omega_{p} t}\right)=$ $\frac{1}{2} E(z, t) e^{-i \omega_{p} t}+c . c$., with the field envelope $E(z, t)=$ $\sum_{k} E_{k} e^{i k z} ; \Phi$ is the ion velocity potential, $v_{i}=\frac{\partial}{\partial z} \Phi ; \omega_{p}$ is the plasma frequency, $T_{e}$ and $T_{i}$ are the electron and ion temperatures; $l_{D}$ is the electron Debye length.
The above equations have a Hamiltonian structure with Hamiltonian

$$
\begin{aligned}
H= & \int d z\left\{\frac{|E|^{2}}{8 \pi}\left(1+\frac{\delta n}{2 n}\right)+\frac{3}{16 \pi}\left|l_{D}^{2} \frac{\partial E}{\partial z}\right|^{2}\right. \\
& \left.+\frac{n T_{e}}{2}\left(\frac{\delta n}{n}\right)^{2}+\frac{n m_{i}}{2}\left(\frac{\partial \Phi}{\partial z}\right)^{2}\right\},
\end{aligned}
$$

and conserve the total number of quanta of the highfrequency (HF) field, i.e. $I_{1}=\int d z|E|^{2} / 8 \pi=$ const, where $L$ is the size of the system. The longitudinal momentum is also conserved

$$
\begin{aligned}
P_{z} & =\int d z\left\{m_{i} \delta n \frac{\partial}{\partial z} \Phi+\frac{i}{16 \pi \omega_{p}}\left(E \frac{\partial}{\partial z} E^{*}-E^{*} \frac{\partial}{\partial z} E\right)\right\} \\
& =\int d z\left\{m_{i} \delta n \frac{\partial}{\partial z} \Phi\right\}+\sum_{k} \frac{k}{\omega_{p}} \frac{\left|E_{k}\right|^{2}}{8 \pi}=\text { const. }
\end{aligned}
$$

With the Hamiltonian $H, \frac{\delta n}{n}$ and $n m_{i} \Phi$ are canonically conjugated variables

$$
\begin{gathered}
\frac{\partial}{\partial t} \frac{\delta n}{n}=\frac{\delta H}{n m_{i} \delta \Phi}=\left(\int d z \frac{\partial \Phi}{\partial z} \frac{\partial \delta \Phi}{\partial z}\right) / \delta \Phi=-n m_{i} \frac{\partial^{2} \Phi}{\partial z^{2}} \\
\frac{\partial \Phi}{\partial t}=-\frac{\delta H}{n m_{i} \delta \frac{\delta n}{n}}=-\left(\frac{|E|^{2}}{16 \pi n m_{i}}+\frac{T_{e}}{m_{i}} \frac{\delta n}{n}\right) .
\end{gathered}
$$

Note that $E e^{-i \omega_{p} t}$ and $E^{*} e^{i \omega_{p} t} / 4 \pi \omega_{p}$ are other canonical variables.

The Hamiltonian description is suitable and allows one to describe the interaction of beam electrons with Langmuir waves by the total Hamiltonian of the Langmuir waves-electron beam-ion sound waves' system $H_{\text {total }}=$ $H+H_{p}$, where $H_{p}$ is the Hamiltonian of the particles describing the $1 \mathrm{D}$ motion of the electrons in the potential field of the waves

$$
\begin{gathered}
H_{p}=\sum_{p}\left(\frac{P_{p}^{2}}{2 m_{e}}-e \operatorname{Re} \sum_{k} \varphi_{k}(t) e^{i k z_{p}}\right) \\
v_{p}=\frac{d z_{p}}{d t}=\frac{\partial H_{p}}{\partial P_{p}}=\frac{P_{p}}{m_{e}}, \quad \frac{d P_{p}}{d t}=-\frac{\partial H_{p}}{\partial z_{p}}, \\
\frac{d P_{p}}{d t}=\frac{d m_{e} v_{p}}{d t}=-e E, E=-\nabla \varphi=\operatorname{Re}\left(-i k \varphi_{k} e^{i k z_{p}-i \omega_{k} t}\right) .
\end{gathered}
$$

with $\varphi_{k}(t)=\varphi_{k} e^{-i \omega_{k} t}$. The model is applicable in the case of a tenuous beam (with density much smaller than the background density, $n_{b} \ll n_{0}$ ), when the interaction of the beam electrons with the Langmuir waves is essential only at the Landau resonances. At the same time it is supposed that the bulk nonresonant plasma electrons 
define the linear properties of the waves. Thus the full Hamiltonian of the system is

$$
\begin{gathered}
H_{\text {total }}=H+H_{p}=H+\sum_{p} \frac{P_{p}^{2}}{2 m_{e}}-e \operatorname{Re} \sum_{k} i \frac{E_{k}}{k} N J_{k}^{*} \\
=H+L n_{b}\left(\left\langle\frac{m_{e} v_{p}^{2}}{2}\right\rangle-e \operatorname{Re} \sum_{k} i \frac{E_{k}}{k} J_{k}^{*}\right)
\end{gathered}
$$

where $J_{k}=\frac{1}{N} \sum_{p} e^{i \omega_{p} t-i k z_{p}} ; N$ is the number of particles; one can write for a moment that $L=1$ to avoid uninteresting details in calculations. Then, calculating $\frac{\partial}{\partial t} E e^{-i \omega_{p} t}=-i \delta H / \delta\left(\frac{E^{*} e^{i \omega_{p} t}}{4 \pi \omega_{p}}\right)$, we obtain the equation of evolution of the HF electric field

$$
\begin{gathered}
i\left(\frac{\partial}{\partial t}+v\right) E+\frac{3}{2} \omega_{p} l_{d}^{2} \frac{\partial^{2}}{\partial x^{2}} E-\frac{\omega_{p}}{2} \frac{\delta n}{n} E \\
=2 \pi e i n_{b} \sum_{k} e^{i k z} \frac{\omega_{p}}{k} J_{k},
\end{gathered}
$$

where $v$ is a damping factor. Of course the set of above equations can be obtained by another way, without using Hamiltonians.

In our simulations we impose periodic boundary conditions. To avoid pitfalls related to such assumption we consider a rather long system $\left(L \geq 5000 l_{d}\right)$; thus the time necessary for beam electrons to cross the simulation box is smaller or comparable to the simulation time. Rewriting the equations in the k-space we get the set of ordinary differential equations $(\rho=\delta n / n)$

$i\left(\frac{\partial}{\partial t}-\gamma_{k}\right) E_{k}=\frac{3}{2} \omega_{p} k^{2} l_{d}^{2} E_{k}+\left(\omega_{p} \frac{\rho}{2} E\right)_{k}+i \frac{2 \pi e \omega_{p} n_{b}}{k} J_{k}$,

which, together with the equations describing the motion of beam electrons, forms the complete set of equations of our model. Here the terms with $\gamma_{k}=\gamma_{k}^{(i)}+\gamma_{k}^{(e)}$ give the possibility to include damping of Langmuir and ion sound waves due to Landau resonance with thermal ions and electrons.

For numerical solution, we rewrite the equations in dimensionless form introducing the new variables $\tau=$ $\omega_{p} t, z \rightarrow z / l_{d}$ and $v_{p}=v_{p} / v_{T_{e}}$ (or $\omega_{p} \rightarrow 1, l_{d} \rightarrow 1$, $\left.v_{T_{e}} \rightarrow 1\right)$

$$
\frac{d z_{p}}{d t}=v_{p}, \quad \frac{d v_{p}}{d t}=-\frac{e}{m_{e} v_{T_{e}} \omega_{p}} \operatorname{Re} \sum_{k} E_{k} e^{i k z_{p}-i \omega_{k} t},
$$

where the electric field is normalized according to $e E_{k} / m_{e} v_{T_{e}} \omega_{p}=E_{k} / E_{*} \rightarrow E_{k}$. In the absence of density fluctuations we have

$$
H_{p}+H_{w}=\frac{n_{b}}{n_{0}}\left(\frac{1}{N} \sum_{p} \frac{v_{p}^{2}}{2}+R e \sum_{k} \frac{E_{k}}{k} i J_{k}^{*}\right)
$$

$$
+\sum_{k}\left|E_{k}\right|^{2}=\text { const }
$$

We use a leap frog scheme for solving the Newton equations and discrete time approximations for the differential equations describing the evolution of the electric fields, the plasma density $\rho$ and velocity $v$ (with Fourier component $u_{k}$ )

$$
\begin{gathered}
\frac{E_{k}^{n+1}+E_{k}^{n}}{\tau}=-i\left(w_{k} \frac{E_{k}^{n+1}+E_{k}^{n}}{2}+\left(\frac{\rho}{2} E\right)_{k}^{n}+i \beta_{k} J_{k}^{n}\right), \\
\rho_{k}^{n+1}=\rho_{k}^{n}-i k c_{s} \tau\left(\frac{u_{k}^{n+1}+u_{k}^{n}}{2}+(\rho v)_{k}^{n}\right), \\
u_{k}^{n+1}=u_{k}^{n} \frac{4-\left(k c_{s} \tau\right)^{2}}{4+\left(k c_{s} \tau\right)^{2}}-\frac{i 4 k c_{s} \tau}{4+\left(k c_{s} \tau\right)^{2}}\left(\rho_{k}^{n}+\frac{1}{4}\left(|E|^{2}\right)_{k}^{n}\right),
\end{gathered}
$$

where $\beta_{k}$ and $w_{k}$ are depending on the waves' properties; $c_{s}$ is the ion sound velocity, $c_{s}^{2}=$ $\left(\left(T_{e}+3 T_{i}\right) / m_{i}\right)\left(1 / v_{T_{e}}\right)^{2}=\left(m_{e} / m_{i}\right)\left(1+3 T_{i} / T_{e}\right) \simeq$ $m_{e} / m_{i}$; we suppose that $T_{i} / T_{e}=0$. Typical initial conditions are : the HF electric fields are presented as a set of waves with small amplitudes and random phases (thermal noise); the electron beam is described by a maxwellian drifting with velocity $V_{b}$ and is supposed to be uniformly distributed in space with $n_{b} \leq 10^{-4} \div 10^{-5} n_{0}$; the initial background density fluctuations verify $\delta n / n_{0} \leq 3 \div 5 \%$ and present non regular profiles with typical scales $\lambda \sim 300 \div 2000 l_{d}$.

\section{SIMULATION RESULTS AND CONCLUSIONS}

The simulation results are presented below in the form of animations (see files online), where all physical quantities are shown using the dimensionless parameters presented above. The first animation (see Anima tion_r23_1.gif online) shows the development of the beam instability in a system with a single density hole of regular shape at $t=0$. The upper panel presents the time evolution of the profiles of wave energy and plasma densities (blue and black lines, respectively). The middle panel shows the wave form of the Langmuir waves (in blue) together with the density variations (in black). On the left bottom panel one can see the evolution of the Langmuir waves' spectrum, whereas the middle bottom panel shows the dynamics of the beam distribution $f\left(v_{z}\right)$ as a function of the electron velocity $v_{z}$ (with the waves' amplitudes in arbitrary units represented by arrows as a function of the phase velocities). On the right bottom panel the evolution of the total energy carried by the Langmuir waves as a function of time is shown. 


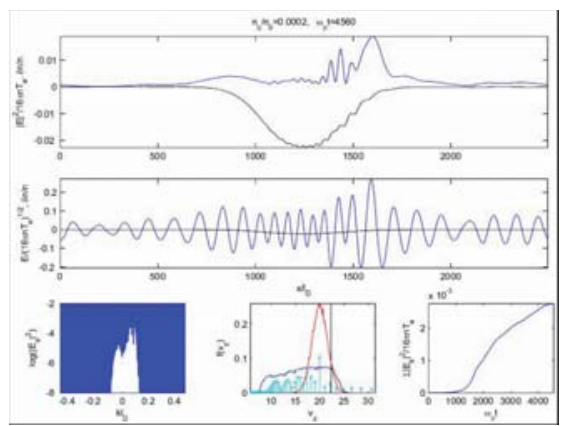

FIGURE 1. Beam instability in a plasma with one density well : profiles of wave energy, electric field and density at $\omega_{p} t=4500$.

One can see how wave packets are formed as a result of wave growth due to beam-plasma instability as well as to wave propagation over the density hole. The simulation demonstrates that only a small part of the wave energy becomes trapped inside the density well, other waves leaving the well and forming narrow packets. A strong relaxation of the beam occurs and a plateau-like velocity distribution of electrons is formed. Because the beam density is rather large $\left(n_{b} / n_{0}=0.0002\right)$, the wave energy grows up to high values at the late stage of the simulation, some modulation instability starts and some solitons are formed. Fig. 1 shows the system's evolution at one moment of time $\left(\omega_{p} t=4500\right)$. The profiles of the wave field amplitudes obtained can be compared with typical waveforms observed by the WIND experiment.

In the second animation (see Animation_r10080_l.gif online), the simulation has been carried out for a long system with quasi-random density fluctuations presenting several wells. The panels represent the variations of the same physical quantities as in the first animation. The only difference is an additional bottom panel showing the growth of the number of electrons accelerated above the velocity $v_{a c c}>V_{b}$. This long time simulation in a long system is performed up to the full relaxation of the beam and allows to conclude that in a plasma with density fluctuations the beam instability of Langmuir waves leads to the following results (see also Animation_r10122_l.gif online) : (1) localized wave packets are formed due to the combination of both effects of wave instability and wave propagation in plasma inhomogeneities, (2) the relaxed beam velocity distribution shows a significantly smaller positive slope which remains positive even at the end of the relaxation whereas the instability is locked, and (3) the relaxation process widens the velocity distribution in the range $V<V_{b}$ and, when the level of density fluctua- tions becomes sufficiently high, i.e.

$$
\sqrt{\left\langle\left(\frac{\delta n}{n_{0}}\right)^{2}\right\rangle} \gg \alpha\left(\frac{v_{T_{e}}}{V_{b}}\right)^{2}
$$

an efficient particle acceleration by waves leads to the formation of a tail in the distribution. High level density fluctuations in the solar wind can be responsible for very long relaxation lengths of beams interacting with Langmuir waves.

\section{ACKNOWLEDGMENTS}

A.V., V.K. and E.K. acknowledge financial support of the Russian Ministery of Education through the grant "Wave-particle interaction in strongly inhomogeneous plasmas of the solar wind"; V.K. acknowledges the financial support of CNES through the grant Cluster Co-I DWP; C.K. acknowledges Dr. Marie Flé for her technical support at the Direction Informatique of the Paris South University.

\section{REFERENCES}

1. R. P. Lin, D. W. Potter, D. A. Gurnett, and F. L. Scarf, Astrophysical Journal 251, 364-373 (1981).

2. D. A. Gurnett, R. R. Anderson, F. L. Scarf, and W. S. Kurth, J. Geophys. Res. 83, 4147-4152 (1978).

3. R. Smith, M. Goldstein, and K. Papadopoulos, Astrophys. J. 234, 348 (1979).

4. L. Muschietti, M. V. Goldman, and D. Newman, Solar Phys. 96, 181-198 (1985).

5. P. Robinson, Phys. Plasmas 2, 1466-1479 (1995).

6. P. A. Robinson, A. J. Willes, and I. H. Cairns, Astrophysical Journal 408, 720-734 (1993).

7. C. R. Boshuizen, I. H. Cairns, and P. A. Robinson, Journal of Geophysical Research (Space Physics) 109, 8101 (2004).

8. D. D. Ryutov, Soviet Journal of Experimental and Theoretical Physics 30, 131 (1970).

9. B. N. Breizman, and D. D. Ruytov, Soviet Journal of Experimental and Theoretical Physics 30, 759 (1970).

10. L. M. Celnikier, C. C. Harvey, R. Jegou, P. Moricet, and M. Kemp, Astron. Astrophys. 126, 293-298 (1983).

11. W. M. Cronyn, Astrophysical Journal 161, 755 (1970).

12. L. M. Celnikier, L. Muschietti, and M. V. Goldman, Astron. Astrophys. 181, 138-154 (1987).

13. P. J. Kellogg, and T. S. Horbury, Annales Geophysicae 23, 3765-3773 (2005).

14. H. A. S. Reid, and E. P. Kontar, Astrophysical Journal 721, 864-874 (2010).

15. A. Zaslavsky, A. S. Volokitin, V. V. Krasnoselskikh, M. Maksimovic, and S. D. Bale, Journal of Geophysical Research (Space Physics) 115, 8103 (2010).

16. A. Zaslavsky, A. S. Volokitin, V. V. Krasnoselskikh, M. Maksimovic, and S. D. Bale, Twelfth International Solar Wind Conference 1216, 296-299 (2010). 